\title{
Practical advices for optimal CT scanner dose in children
}

\author{
F. Malchair and C. Maccia* \\ CAATS, 119-121, Grande Rue, 92310 Sèvres, France.
}

Received: 3 April 2020 / Accepted: 15 April 2020

\begin{abstract}
Radiation protection and dose optimisation in computerized tomography (CT) for pediatric patients are of utmost importance because of the potential risk of cancer induction by exposure to ionizing radiation. A review of available technical features in modern CT machines aiming at reducing and/or optimizing patient dose was performed. Practical advices to operators were listed according to dose-related technical solutions studied to decrease the doses and to practically implement dose optimisation.
\end{abstract}

Keywords: CT / patient dose optimisation / radiation protection / pediatric imaging

\section{Introduction}

Doubts exist on the possible increase in the number of cancers (brain cancers, leukaemia and others) following computerized tomography (CT) irradiation of children (Brenner et al., 2001; Pearce et al., 2012; Kritsaneepaiboon et al., 2012; Mathews et al., 2013; Miglioretti et al., 2013; Meulepas et al., 2019; Shao et al., 2020).

Final results of the European EPI-CT epidemiologic survey in about one million children are pending while details of the methodology (Bosch de Basea et al., 2015) and cohort profile (Bernier et al., 2019) have already been published.

In view of these recent publications and of the known sensitivity to radiation of children, a very cautious approach has to be taken for pediatric $\mathrm{CT}$ examinations. Furthermore, the longer life expectancy of children gives them a greater probability to develop a cancer which is an age related process. Moreover the smaller amount of fat that separate organs from each other in children contributes to a larger absorbed dose for the same technical parameters and displayed CT dose index (CTDIvol) in comparison to adults. Indeed the principles of radiological protection of the International Commission of Radiological Protection have to be enforced in children (ICRP, 2007):

- all CT examinations must be justified (Sodhi et al., 2015);

- all CT examinations, whether performed in a children hospital, in a general hospital or in a private practice, must be optimized and the parameters scaled to the individual child size (Strauss et al., 2010; Mannudeep, 2016) to obtain a quality sufficient to allow the precise diagnosis;

- the principle of dose limitation does not apply to patients.

Therefore the optimal CT scanner dose is entirely in the hands of the radiological team (radiologist, radiographer,

*Corresponding author: carlo.maccia@caats.fr medical physicist, application engineer etc...) who must choose the optimized parameters for the lowest possible dose to achieve an appropriate diagnosis for each child. It is worth recalling that medical exposures are intentional exposures and that each $\mathrm{CT}$ scanner examination must be justified, i.e., a priori useful in the following sense: " $A$ useful investigation is one in which the result-positive or negative - will alter management (of the patient) or add confidence to the clinician's diagnosis" (Radiation Protection 118, 2000).

Thus this paper reviews CT scanner parameters and protocols that must be used for optimal CT dose in children. This paper addresses:

1 How to decrease CT doses?

2 How to practically implement dose optimization?

\section{How to decrease CT doses?}

Various technical possibilities are available in modern CT machines to reduce patient dose in CT pediatric examinations.

\subsection{Fixed tube current (technique charts)}

Due to the normalized nature of CT data which ensures that the image always appears properly exposed, CT users are not technically compelled to decrease the tube-current-time product (mAs) for small patients, which may result in excess radiation dose for them.

As with radiographic and fluoroscopic imaging, the operator should be provided with appropriate guidelines for mAs selection as a function of patient size. These are often referred to as technical charts. In CT, the tube current exposure time and tube potential can be altered to give the appropriate exposure to the patient. 
Once users have standardized the tube potential $(\mathrm{kV})$ according to patient weight, and gantry rotation time (s) is set for a given clinical application, the manner in which $\mathrm{mA}$ should be adjusted as a function of patient size should be related to the overall attenuation, or thickness, of the anatomy of interest as opposed to patient weight.

\subsection{Tube current (mA) modulation}

Wide variations in patient absorption occur both with projection angle and anatomic region and are not considered when using a fixed tube current.

The projection with the most noise primarily determines the noise of the final image. Hence, data acquired through body parts having less attenuation can be acquired with substantially less radiation without impairing the final image quality (Radiation Technology Skills UP Series, 2019).

- Angular (x,y) mA modulation

Angular (x,y) $\mathrm{mA}$ modulation deals with the variation in $\mathrm{X}$-ray attenuation around the patient by varying the $\mathrm{mA}$ as the $\mathrm{X}$-ray tube rotates around the patient (e.g., in the Antero Posterior versus Lateral direction).

- Longitudinal (z) mA modulation

Longitudinal (z) mA modulation deals with the varying attenuation of the patient among anatomic regions by varying the $\mathrm{mA}$ along the $\mathrm{z}$-axis of the patient (e.g., shoulders versus the abdomen).

- Angular and longitudinal (x,y,z) mA modulation

Angular and longitudinal (x,y,z) mA modulation combines the previous two methods to vary the $\mathrm{mA}$ both during rotation and along the z-axis of the patient.

\subsection{Automatic exposure control (AEC)}

For CT systems it is technologically possible to adjust the X-ray tube current in real time in response to variations in X-ray intensity at the detector. These methods of adapting the tube current to patient attenuation, known generically as automatic exposure control (AEC), are analogous to phototiming in general radiography and have demonstrated reductions in dose of about $20-40 \%$ when image quality is appropriately specified (Martin and Sookpeng, 2016). Among all these techniques, the major issue is the definition of the image quality which differs depending on CT manufacturers:

- GE Healthcare uses a concept known as the Noise Index;

- Canon (formerly Toshiba) allows two ways to prescribe image quality in their Sure Exposure AEC algorithm: Standard Deviation and Image Quality Level;

- Philips Medical System uses a Reference Image from a satisfactory patient exam (Reference Case) stored in the system;

- Siemens Healthineers uses a Quality Reference mAs to define the effective $\mathrm{mAs}(=\mathrm{mAs} / \mathrm{pitch})$ required to produce a specific image quality in an $80 \mathrm{~kg}$ patient $(20 \mathrm{~kg}$ for pediatric cases) for a given protocol.
It is important to know how the scanner operates in order to fully understand how to set the mA modulation system (Martin and Sookpeng, 2016).

\subsection{Adjusting kV based on patient size}

The principle behind the benefit of lower $\mathrm{kV}$ is that in many CT exams involving the use of iodinated contrast media, the superior enhancement of iodine at lower tube potentials improves the visualisation of hypervascular or hypovascular pathologies.

Despite the increased noise level, the quality of images of small sized patients is better than that obtained with higher tube potential, for the same radiation dose (if $\mathrm{mA}$ is increased) or much lower.

Some manufacturers offer an assistance for choosing $\mathrm{kV}$ based on the radiographic view (Li et al., 2017).

\subsection{Dose and Pitch relationship}

Pitch in the multidetector, spiral CT era is defined as table travel per rotation divided by beam collimation.

A pitch equal to 1 means neither overlap nor gap between slices. A pitch $>1$ results in a lower radiation dose (and lower sampling). A pitch $<1$ results in an increased dose (and overlap of anatomy).

It is worth knowing that, through an automatic proportional increasing of tube current when pitch increases, some CT brands can provide an increased radiation dose values for smaller pitch selections in order to avoid any image quality impairment.

\subsection{Detector configuration, slice thickness and collimation}

The detector configuration should be determined on the basis of the type of study performed. For routine acquisitions, without the need for thin-section 3-D imaging, there is no need for extremely thin effective detector thicknesses.

In such cases, if thick-section 5-mm images in the axial, coronal, and sagittal planes are sufficient for radiologist review, the effective detector thickness can be widened to 1.25 or $1.5 \mathrm{~mm}$ (rather than 0.75 or $0.5 \mathrm{~mm}$ ), keeping a similar image quality.

However, if thin-section multiplanar reconstructions (MPRs) or high-resolution 3-D images (with increased spatial resolution in the $\mathrm{z}$-axis) are required, a thin effective detector thickness $(0.5-0.75 \mathrm{~mm})$ may be necessary, although there is an associated dose penalty.

\subsection{Adaptive collimation}

In spiral CT, an extra half-rotation of the gantry before and after each scan is routinely done, fully irradiating the detector throughout, even though only part of the acquired data is necessary for image reconstruction. As a result, the wide cone beam exposes tissue that will never be part of reconstructed images. 
The adaptive collimation, a technology based on precise, fast, and independent movement of both collimator blades, limits this over-ranging.

The pre-patient collimator asymmetrically opens and closes at the beginning and end of each spiral scan, temporarily blocking those parts of the X-ray beam that are not used for image reconstruction. As a result, only the targeted tissue is irradiated, since adjacent organs at risk are protected.

By using adaptive section collimation, a substantial dose reduction of up to $10 \%$ can be achieved for cardiac and chest. For scan ranges smaller than $12 \mathrm{~cm}$ such dose reduction can reach 40\%. (Deak et al., 2009).

\subsection{Spectral imaging}

Spectral imaging is a way to visualize tissue composition. Dual energy imaging allows a reduction in radiation dose by eliminating the need for true non-contrast imaging and reducing the need for repeat exams. Lower energy images can be used to salvage bad CT angiograms, do low-contrastlow-dose exams and increase conspicuity of lesions among other things.

The two dual-energy CT postprocessing techniques that best exploit the advantages of dual-energy $\mathrm{CT}$ in children are the material-decomposition images (which include virtual nonenhanced, iodine, perfused lung blood volume, lung vessel, automated bone removal, and renal stone characterization images) and virtual monoenergetic images (Siegel and Ramirez-Giralodo, 2019).

\subsection{Artificial Intelligence (AI)}

Although AI is only at the beginning of its full potential, it has potential for many applications especially in oncology in tumour diagnosis (early detection, flagging of abnormal findings, differentiation between benign and malignant tumours, vessel involvement, tumour staging...) (DaldrupLink, 2019).

Machine learning has the potential to vastly advance medical imaging, particularly computerized tomography (CT) scanning, by reducing radiation exposure and improving image quality (Rensselaer Polytechnic Institute, 2019).

It will help in adapting and choosing in the most appropriate way exposure parameters and protocols according to diagnostic objectives that doctors are seeking for.

\subsection{Organ sparing options}

Organ sparing refers to selective reduction of radiation exposure of dose-sensitive anatomical regions such as female breasts, eye lenses, and thyroid.

Unfortunately, protection shields give rise to artefacts that may prevent their use in specific cases (Liao et al., 2019).

Alternatively, organ dose modulation systems are organ sparing options that allow to reduce or completely switch off tube current during the rotation phase in which the concerned anatomical regions are most directly exposed to radiation, making a dose reduction of individual anatomical regions possible (Kim et al., 2017).

\subsection{Reconstruction algorithms-Iterative reconstruction (IR)}

Model-based iterative reconstruction refers to iterative algorithms used to reconstruct $2 \mathrm{D}$ and $3 \mathrm{D}$ images. In CT an image must be reconstructed from projections of an object. Iterative reconstruction techniques are a better alternative to the common filtered back projection (FBP) method, which directly calculates the image from raw data in a single reconstruction step.

Iterative reconstruction techniques have demonstrated the potential for improving image quality thus allowing a reduction in radiation dose (Khawajo et al., 2014; Brand et al., 2018).

The first generation used only image correction in addition to image data reconstruction (image space) and allowed up to $40 \%$ gain in dose.

The most widely used second generation uses raw data reconstruction as well as image data reconstruction and image correction using system noise statistics in an iterative way (image and raw data space) allowing for very fast reconstruction and up to $60-70 \%$ decrease in dose.

So far, the third generation of iterative reconstruction takes minutes as compared to seconds in other IR and consequently is not used in routine practice. It uses the system noise statistics and a mathematical model of the scanner allowing for extremely low doses, comparable to conventional X-ray doses.

Metal artefact algorithm may also help in avoiding unnecessary repeated exams.

Artificial intelligence will potentially further increase the performance of reconstruction methods (Willemink and Noël, 2019).

\subsection{Diagnostic Reference Levels (DRL)}

Although DRL do not decrease dose by themselves, they are an interesting tool for optimization. The need to establish national DRLs as well as local DRLs is highlighted in many recent publications (Radiation Protection 185, 2018; Roch et al., 2018).

Local DRLs are defined as the third quartile value of a set of dosimetric and morphologic data from local patients for defined CT procedures and for defined patient weight range. It would make more sense to define DRL for specific clinical indications of CT procedures (Radiation Protection 185, 2018; Granata et al., 2019).

National DRLs are based on the 3rd quartile of the distribution from all local medians (Journal officiel de la république française, 2019).

European DRLs are based on the distribution of national DRLs.

There are many challenges in defining pediatric DRLs: categorizing according to weight rather than age is better but more difficult to achieve.

\section{How to practically implement dose optimization?}

As a general rule, dose reduction in $\mathrm{CT}$ is based on the following principles, valid for each procedure: 
All technical parameters of the scan must be adapted ideally to the patient body mass index, if not to the weight and failing that to the patient age, to the organ and to the volume of the organ to be examined, its natural contrast, the size of the lesion, and the indication of the examination.

\subsection{Concerning the equipment}

- When buying a CT scanner, take the dosimetry criterion described above into account along with the other criteria (cost, ergonomy, throughput, etc...). This should contribute to progressively get rid of the machines that require the largest amounts of dose.

- Have preventive maintenances and quality control tests done, to ensure conformity and performance stability of the machine.

- Make sure the complete dose data of Radiation Dose Structured Report (RDSR) are sent to a dose server - Dose Archiving and Communication System (DACS) - allowing dose traceability, analysis of dose characteristics, assessment of local DRLs for the whole pediatric population thus contributing to the optimisation through temporal analysis of data as well as the required local DRLs that are usually based on a limited set of patients' data.

\subsection{Concerning CT protocols}

- Position the patient carefully, check that he/she is at the isocenter of the scanner, that the arms are correctly positioned in order to avoid beam hardening artefacts and dose increase if $\mathrm{mA}$ modulation is used, that he/she is quiet enough and will not move during the examination. Incorrect positioning may result in significant (up to 25\%) dose increase (Raman et al., 2013; Zacharias et al., 2013).

- Prefer a Postero Anterior radiographic view using the lowest possible $\mathrm{kV}$ and $\mathrm{mA}$ and adapt as much as possible the field of view to the patient body size.

- Use the smallest possible scan range that is compatible with the scan request. Limit the scanned volume (field of view, number of slices) to what is just necessary to answer the clinical questions justifying the procedure (Raman et al., 2013; Zacharias et al., 2013)

- Choose the lowest kV compatible with the necessary image quality according to patient weight. For the same field of view, dose to patient increases with tension to the power of 2.5. In practice, the $\mathrm{kV}$ used for pediatric CT are between 70 and $120 ; 70$ to $80 \mathrm{kV}$ being used for less than $30 \mathrm{~kg}$ patients, $100 \mathrm{kV}$ for patients until $60 \mathrm{~kg}, 120 \mathrm{kV}$ being used for adult sized children. For thorax CT it is never useful to use more than $100 \mathrm{kV}$.

- Lower the dose by decreasing current $\mathrm{mA}$ and/or rotation time within limits that are compatible with the image quality criteria (Signal to Noise ratio for instance). The dose delivered to the patient is directly proportional to $\mathrm{mAs}$, and an overexposure (mAs in excess) can be totally overlooked. The mAs can be decreased for smaller morphotype with no significant change of the image quality. Surveys have shown that CT parameters are not adapted enough to body built of patients.

- Be careful when selecting thin slices $(<1 \mathrm{~mm})$ because dose is automatically increased when collimation decreases to these levels.

- Do not use gantry tilt especially for head CT: the helical mode allows for multiplanar reconstructions (MPR) that show images in the coronal plane while avoiding exposure of the lens of the eyes.

- Both collimation (function of size and number of detector arrays) and reconstructed slice thickness must be chosen at the time of acquisition. Acquisition parameters must be chosen in function of the desired reconstruction slice thickness in order to obtain the required image quality.

- Use the manufacturer's pediatric protocols, age or weight classified, they can help choosing the best parameters considering the child's morphotype. Be aware that these protocols still have to be adapted as a function of the clinical indication.

- Use automatic current modulation systems (mA modulation) since they allow for dose reduction. These techniques must be used in addition to a previous optimization of the protocols. Quality indices (noise index in the image, reference $\mathrm{mAs}$ or reference image according to different manufacturers) are used to maintain a constant image quality.

- Shields can be used to protect sensitive organs. Bismuth shields are available commercially: breast shields for using during the thorax examination of young females, thyroid shields, etc... They reduce the absorbed dose by a factor of 2 but create artefacts in the image. Their use is optimized by putting foam between the surface of the skin and the shielding. Their use is not recommended when $\mathrm{x}-\mathrm{y} \mathrm{mA}$ modulation is used, and they must be put in place after the radiogram has been done (risk of overexposure). Alternatively, organ dose modulation can be used to spare organs at risk.

- Do not use lead garments to protect radiosensitive organs (thyroid, pelvis) because they might negatively interfere with $\mathrm{mA}$ modulation and resulting in a fault sense of protection (Marsh and Silosky, 2019).

\section{Conclusion}

Medical exposures constitute a priority in radiological protection and all efforts need to be made to control the growth of the doses due to diagnostic examination with ionizing radiation especially for CT (Bourguignon et al., 2017). The most important issues in paediatric imaging are to get images of good quality for diagnosis and to avoid repeating the examination.

We have investigated in this paper all possible ways to optimize doses in pediatric CT. Methods reviewed in this manuscript are useful for the optimisation process in order to get a diagnostic image quality with the lowest patient exposure.

To achieve that in practice, a voluntary attitude of radiologists is necessary to really optimize the technical parameters of the examinations. 
Certainly the assessment of DRLs for each CT equipment and in each institution constitutes a simple way to control the efficacy of the optimization.

It is important to create a core team for patient dose management consisting of radiologists, medical physicists, technicians as recommended by the new EURATOM directive in order to evaluate the optimisation process.

Training of the operators on patient radiation protection issues is of utmost importance including training on how to use the CT scanners safely and how to use the available dose/image quality methods.

\section{References}

Bernier MO, Baysson H, Pearce MS, Moissonnier M, Cardis E, Hauptmann M, Struelens Dabin J, Johansen C, Journy N, Laurier D, Blettner M, Le Cornet L, Pokora R, Gradowska P, Meulepas JM, Kjaerheim K, Istad T, Olerud H, Sovik A, Bosch de Basea M, Thierry-Chef I, Kaijser ML, Nordenskjöld A, Berrington de Gonzalez A, Harbron RW, Kesminiene A. 2019. Cohort profile: the EPI-CT study: a European pooled epidemiological study to quantify the risk of radiation-induced cancer from paediatric CT. Int. J. Epidemiol. 48(2): 379-381. https://doi.org/10.1093/ije/ dyy 231.

Bosch de Basea M, Pearce MS, Kesminiene A, Bernier MO, Dabin J, Engels H, Hauptmann M, Krille L, Meulepas JM, Struelens L, Baatout S, Kaijser M, Maccia C, Jahnen A, Thierry-Chef I, Blettner M, Johansen C, Kjaerheim K, Nordenskjöld A, Olerud H, Salotti JA, Andersen TV, Vrijheid M, Cardis E. 2015. EPI-CT: design, challenges and epidemiological methods of an international study on cancer risk after paediatric and young adult CT. J. Radiol. Prot. 35: 611.

Bourguignon M, Bérard P, Bertho JM, Farah J, Mercat C, Radioprotection Editorial Board. 2017. What's next in Radioprotection? Radioprotection 52(1): 21-28.

Brand M, Wuest W, May M, Uder M, Sommer M. 2018. Influence of risk-organ-based tube current modulation on CT-induced DNA double-strand breaks in a biological phantom model. J. Radiat. Res. 59(6): 692-699.

Brenner D, Elliston C, Hall E, Berdon W. 2001. Estimated risks of radiation-induced fatal cancer from pediatric CT. AJR Am. J. Roentgenol. 176(2): 289-296.

Daldrup-Link H. 2019. Artificial intelligence applications for pediatric oncology imaging. Pediatr. Radiol. 49(11): 1384-1390.

Deak PD, Langner O, Lell M, Kalender WA. 2009. Effects of adaptive section collimation on patient radiation dose in multisection spiral CT. Radiology 252(1).

Granata C, Sorantin E, Seuri R, Owens CM. 2019. European Society of Paediatric Radiology computed tomography and dose task force: European guidelines on diagnostic reference levels for paediatric imaging. Pediatr. Radiol. 49: 702-705.

ICRP. 2007. The 2007 Recommendations of the International Commission on Radiological Protection. ICRP Publication 103. Ann. ICRP 37(2-4).

Journal officiel de la république française (30 Mai 2019). 2019. Arrêté du 23 mai 2019 portant homologation de la décision no 2019-DC0667 de l'Autorité de sûreté nucléaire du 18 avril 2019 relative aux modalités d'évaluation des doses de rayonnements ionisants délivrées aux patients lors d'un acte de radiologie, de pratiques interventionnelles radioguidées ou de médecine nucléaire et à la mise à jour des niveaux de référence diagnostiques associés.
Khawajo RDA, Singh S, Otrakji A, Padole A, Lim R, Nimkin K, Westra SJ, Kalra MK, Gee M. 2014. Dose reduction in pediatric abdominal CT: use of iterative reconstruction techniquesacross different CT platforms. Pediatr. Radiol. 45.

Kim JS, Kwon SM, Kim JM, Yoon SW. 2017. New organ-based tube current modulation method to reduce the radiation dose during computed tomography of the head: evaluation of image quality and radiation dose to the eyes in the phantom study. Radiol. Med. 122(8): 601-608.

Kritsaneepaiboon S, Trinavarat P, Visrutaratna P. 2012. Survey of pediatric MDCT radiation dose from university hospitals in Thailand: a preliminary for national dose survey. Acta Radiol. 53: 820-826.

Li M, Feng S, Wu N, Zhang L. 2017. Scout-based automated tube potential selection technique ( $\mathrm{kV}$ Assist) in enhanced chest computed tomography: effects on radiation exposure and image quality. J. Comput. Assist. Tomogr. 41(3): 442-445.

Liao YL, Lai NK, Tyan YS, Tsai HY. 2019. Bismuth shield affecting CT image quality and radiation dose in adjacent and distant zones relative to shielding surface: a phantom study. Biomed. J. 43: 343-51.

Mannudeep K. 2016. Optimization of paediatric CT. Phys. Med. 32: 182.

Marsh RM, Silosky M. 2019. Patient shielding in diagnostic imaging: discontinuing a legacy practice. AJR 212: 755-757.

Martin CJ, Sookpeng S. 2016. Setting up computed tomography automatic tube current modulation systems. J. Radiol. Prot. 36: 74-95.

Mathews JD, Forsythe AV, Brady Z, Butler MW, Goergen SK, Byrnes GB, Giles GG, Wallace AB, Anderson PR, Guiver TA, McGale P, Cain TM, Dowty JG, Bickerstaffe AC, Darby SC. 2013. Cancer risk in 680000 people exposed to computed tomography scans in childhood or adolescence: data linkage study of 11 million Australians. BMJ 346: 2360.

Meulepas JM, Ronckers CM, Smets AMJB, Nievelstein RAJ, Gradowska P, Lee C, Jahnen A, van Straten M, de Wit MY, Zonnenberg B, Klein WM, Merks JH, Visser O, van Leeuwen FE, Hauptmann M. 2019. Radiation exposure from pediatric CT scans and subsequent cancer risk in the Netherlands. JNCI111(3): 256-263.

Miglioretti DL, Johnson E, Williams A, Greenlee RT, Weinmann S, Solberg LI, Feigelson HS, Roblin D, Flynn MJ, Vanneman N, Smith-Bindman R. 2013. The use of computed tomography in pediatrics and the associated radiation exposure and estimated cancer risk. JAMA Pediatr. 67: 700-7.

Pearce MS, Salotti JA, Little MP, McHugh K, Lee C, Kim KP, Howe NL, Ronckers CM, Rajaraman P, Sir Craft AW, Parker L, Berrington de González A. 2012. Radiation exposure from CT scans in childhood and subsequent risk of leukaemia and brain tumours: a retrospective cohort study. Lancet 380(9840): 499-505.

Radiation Protection 118. 2000. Referral guidelines for medical imaging. European Commission. Directorate-General for the Environment.

Radiation Protection 185. 2018. European guidelines on diagnostic reference levels for paediatric imaging. https://doi.org/10.2833/ 003998.

Radiation Technology Skills UP Series: Standardization in X-Ray CT Imaging-GALACTIC-(Second edition): Guideline for All About CT exams: Imaging Concept, Ohmsha Ltd, 3-1 Kanda Nishikcho, Chiyoda-ku, Tokyo, 101-8460, Japan. 2019. The Radiation imaging section of the Japanese Society of Radiological Technology.

Raman SP, Mahadevappa M, Blasko RV, Fishman EK. 2013. CT scan parameters and radiation dose: practical advice for radiologists. JACR 10: 840-6. 
Rensselaer Polytechnic Institute. 2019. "Machine learning approach for low-dose CT imaging yields superior results: Findings make a strong case for harnessing the power of artificial intelligence in CT". ScienceDaily. www.sciencedaily.com/releases/2019/06/ 190610111505.htm.

Roch P, Célier D, Dessaud C, Etard C. 2018. Les niveaux de référence diagnostiques en France: une perception contrastée face à un dispositif perfectible mais efficace. Radioprotection 53(1): 13-19.

Shao YH, Tsai K, Kim S, Wu YJ, Demissie K. 2020. Exposure to tomographic scans and cancer risks. JNCI Cancer Spectrum 4(1): pkz072.

Siegel MJ, Ramirez-Giralodo JC. 2019. Dual-energy CT in children: imaging algorithms and clinical applications. Radiology 291(2): 286-297. https://doi.org/10.1148/radiol.2019182289.
Sodhi KS et al. 2015. Clinical application of "Justification" and "Optimization" principle of ALARA in pediatric CT imaging: "How many children can be protected from unnecessary radiation?" Eur. J. Radiol. 84: 1752-1757.

Strauss K et al. 2010. Image gently: ten steps you can take to optimize image quality and lower CT dose for pediatric patients. AJR 194 (4): 868-873.

Willemink MJ, Noël PB. 2019. The evolution of image reconstruction for CT - from filtered back projection to artificial intelligence. Eur. Radiol. 29: 2185-2195.

Zacharias C, Alessio AM, Otto RK, Iyer RS, Philips GS, Swanson JO, Thapa MM. 2013. Pediatric CT: strategies to lower radiation dose. AJR 200: 950-6.

Cite this article as: Malchair F, Maccia C. 2020. Practical advices for optimal CT scanner dose in children. Radioprotection 55(2): 117-122 